- Screen all caseload MND patients for suitability to use ECAS and carry out screens. MND Association (MNDA) provided support with a small grant and resources.

All MND patients were assessed during MDT meetings/ other clinical encounters. Offers of the screen (including written information) were made and ECAS was facilitated by the most appropriate clinician.

Results There were 58 MND caseload patients during 1.9.2016-30.10.2017:

- $10(17 \%)$ completed ECAS

- 25 (43\%) were offered ECAS (3 declined; 20 were awaiting decisions/appointments at the time of submission; 1 did not attend the appointment; 1 did not proceed during the appointment)

- $22(38 \%)$ were not considered (2 did not speak English, 7 died before discussion, 13 were too ill/severely cognitively impaired)

ECAS was facilitated by doctors, physiotherapists and occupational therapists in clinics, care homes and homes. Education activities included a dedicated teaching session and dissemination of MNDA booklets and ECAS.

Discussion ECAS worked best in patients with good insight who were motivated to improve their management. The survey is long to complete and dedicated time was need for completion, usually outside regular clinics. Cognitive impairment is a sensitive topic and the introduction of ECAS was most successful following a cue from a patient or after building a trusting rapport before the introductory discussion. Use of ECAS has triggered the hospice collaboration in ongoing national research of the tool.

\section{TRANSFERRING PATIENTS WITH PALLIATIVE CARE NEEDS - WHICH AMBULANCE SERVICE SHOULD YOU USE?}

E Presswood, E O'Brien, N Pease. Wales Deanery, Velindre Cancer Centre, Welsh Ambulance Service Trust

\subsection{6/bmjspcare-2018-ASPabstracts. 173}

Background Transferring patients with palliative care needs between care settings via the Welsh Ambulance Service Trust (WAST) is a daily component of delivering palliative care. We hypothesised that patients would have quicker and more appropriate ambulance transfers if healthcare professionals working in palliative care had a better knowledge of the ambulance services available; including the pilot of the new End-of-Life Rapid Transport Service.

Method We sent an electronic questionnaire to 236 healthcare professionals working in palliative care in Wales. We tested the respondent's knowledge of the available services provided by WAST and asked respondents to share their experiences of transferring patients via the ambulance service.

Results We had 52 responses to the questionnaire (response rate $22 \%$ ). Respondents were predominantly palliative medicine consultants, registrars and clinical nurse specialists. There was a lack of knowledge about the WAST's ability to transfer patients with syringe drivers and the services ability to make decisions regarding not commencing cardio-pulmonary resuscitation. The Urgent Care Service was not well understood and only $12 \%$ of respondents could easily find the correct contact details for each service.
We created and publicised an aide-memoire (attached) about the different ambulance services available in Wales. It contains information tailored to palliative care health care professionals. The aide memoire is designed to be easily accessible in paper and electronic format.

Conclusion Palliative care healthcare professionals lack sufficient knowledge of the ambulance services available in Wales. The aide memoire we created contains the relevant information that is necessary to make the best use of the ambulance services available. The aide memoire is being used across Wales to improve the use of the ambulance service for patients with palliative care needs.

\section{DELIVERING CANCER PATIENT INFORMATION THROUGH SOCIAL MEDIA: WHOSE BENEFIT?}

${ }^{1}$ Nikki Pease, ${ }^{2}$ Simon Noble. 'Velindre Hospital Cardiff, Velindre Cancer Centre; ${ }^{2}$ Cardiff University

10.1136/bmjspcare-2018-ASPabstracts.174

Introduction Historically, access to cancer information has been limited to printed literature and discussions with healthcare professionals. Opportunities through social media and digital technology have seen notable success in adolescent and young adult health care. Its application in the adult cancer population has not been evaluated.

Objectives To identify the extent cancer patients engage with social media and explore its potential for providing access to oncology information.

Method Patients/carers attending a UK cancer centre were surveyed about their access to and use of social media. Its application to their healthcare support needs was explored with specific attention to their information media preferences. Nonpatient identifiable data, based on a prepared questionnaire were collected via hand held tablets and collated via Survey Monkey.

Results 430 participants, comprising 64\% patients and 36\% carers/ spouse, completed the survey. Median age was 65 year old: $82 \%$ were over 50 and $29 \%$ over $70.16 \%$ of patients did not access the Internet whilst $61 \%$ used it daily. Only 13\% used Twitter, 48\% Facebook and 41\% YouTube.

Only $8 \%$ expressed a preference to receive information by digital media, with the majority favouring face-to-face contact with a healthcare professional $(54 \%)$ or printed media (18\%). Talking to other patients or attending patient support groups were the preference of $11 \%$ and $3 \%$ of participant's respectively. $6 \%$ would seek information themselves using a search engine or visiting of government health website.

Conclusion The majority of patients attending a regional cancer are beyond retirement age with less than $18 \%$ under 50 years old. Whilst most have access to the Internet, engagement with Facebook, Twitter and You Tube is uncommon. This suggests a limited role for social

\section{DEVELOPING AN ENHANCED SUPPORTIVE CARE SERVICE FOR WOMEN SUFFERING FROM THE ADVERSE EFFECTS OF CANCER AND ITS TREATMENT}

Katie Taroni, Helen Reeves, Katherine Hill. St. Giles Hospice

10.1136/bmjspcare-2018-ASPabstracts. 175 